\title{
ABUNDANCES IN THE GALACTIC CLUSTERS OF HYADES AND M 67
}

\author{
R. FOY \\ Observatoire de Meudon, France
}

\begin{abstract}
Two Hyades dwarfs and two giants in the old galactic cluster M 67 have been carefully analyzed from $7 \AA \mathrm{mm}^{-1}$ taken at OHP with an electronic camera. Measurements of weak lines lead to $\ni$ the conclusion that both these clusters have a solar chemical composition, although they have very different ages.
\end{abstract}

To know the global metal abundance of Hyades stars is essential, at least for two reasons: firstly to derive an accurate age for this cluster from theoretical evolutionary tracks; secondly to have a reliable calibration of chemical composition for photometric or low resolution spectroscopic works. So abundance determinations of the Hyades are very numerous. I would want to add another one which will be convincing, I hope, that the metal content of the Hyades is not significantly different from the solar one.

I have studied the two solar type dwarfs of the Hyades cluster HD $28068(=63)$ and HD 28344 (=73), from spectra taken by R. Cayrel at the Observatoire de Haute-Provence with an échelle spectrograph and a Lallemand electronic camera. The dispersion of the spectra is $6 \AA \mathrm{mm}^{-1}$. Spectra of Vesta have been taken in the same way to check equivalent width measurements.

These two dwarfs have been analyzed using Peytremann's model atmospheres. From curves of growth well defined in their weak line part, it comes for the two stars the iron abundance $\left[\frac{\mathrm{Fe}}{\mathrm{H}}\right] \underset{\odot}{\mathrm{H} y \mathrm{ades}} \simeq+0.05$, relative to the Sun.

This abundance is strongly confirmed by the diagram of Figure 1. HD 28068 has the same temperature as the Sun, from colours or from ionization equilibrium. Therefore, differences in equivalent widths of weak lines between HD 28068 and the Sun have to be interpreted in terms of difference in abundance. As shown in Figure 1, the systematic difference in equivalent widths between HD 28068 and Vesta is very small. This proves, by a way independent from atmosphere models, that the Hyades have a solar metal content: it is not possible to assume values of the metal content such as +0.20 or +0.30 for HD 28068. Note that adopting a slightly different effective temperature does not change this conclusion.

I have also analyzed eight giants belonging to the cluster or to the moving group of the Hyades. The mean abundance I have obtained for these giants: $<\left[\frac{\mathrm{Fe}}{\mathrm{H}}\right]_{\odot}^{\mathrm{Hyades}}>=+0.06$ agrees very well with the abundance determined for the two Hyades dwarfs. This good agreement gives confidence in the reliability of abundances determined from the used model atmospheres of giants.

I have therefore applied them to study two giants in the old galactic cluster M 67. I have 


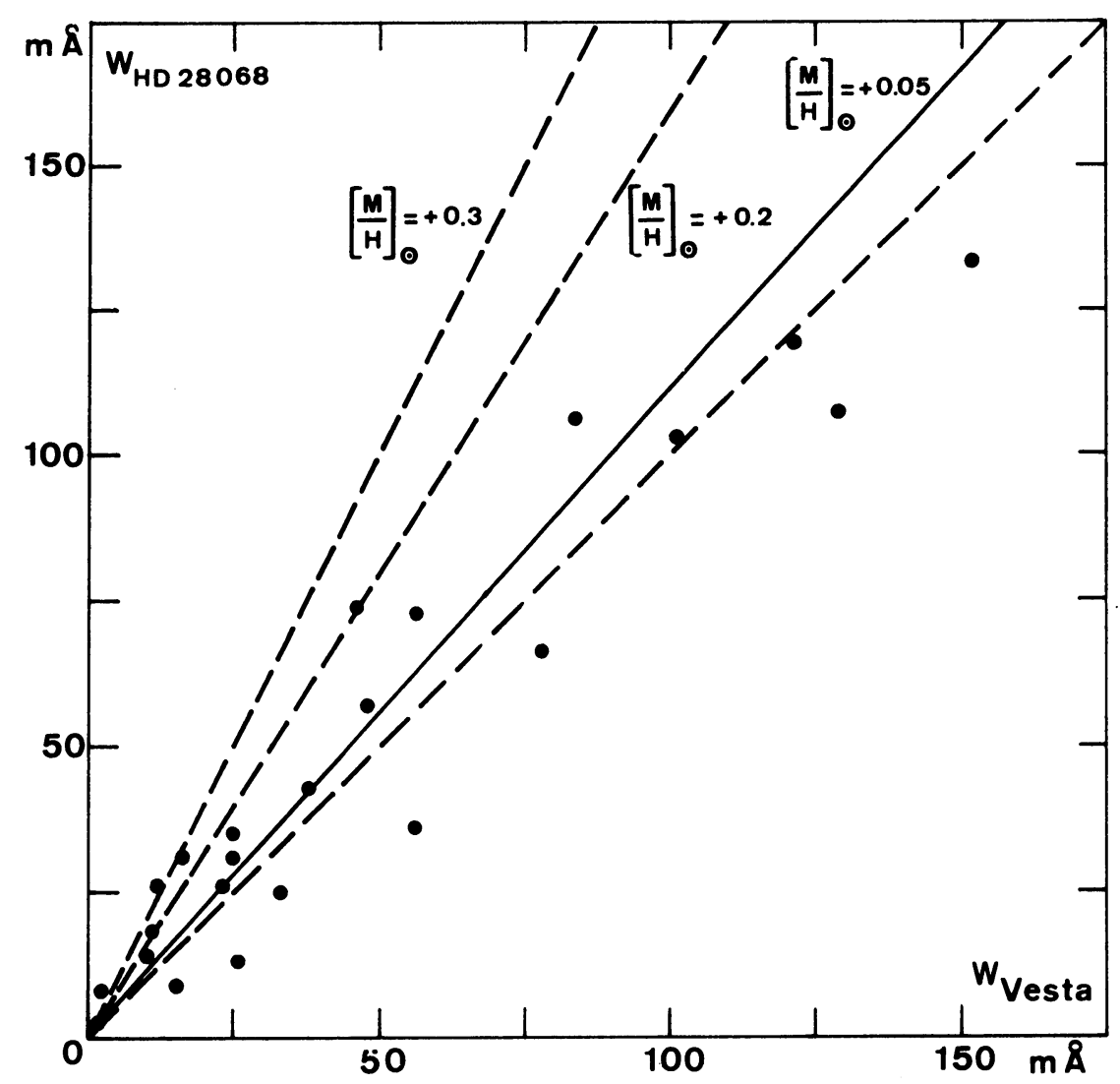

Fig. 1.

chosen them not too bright in order to avoid criticism about non-LTE effects. These stars are F 170 and F 244. I have taken five spectra at the Observatoire de Haute-Provence, with the échelle spectrograph and the Lallemand electronic camera at the Coudé focus of the $1.52 \mathrm{~m}$ telescope. The dispersion was 6 to $8 \AA \mathrm{mm}^{-1}$.

From the well-defined curve of growth of $\mathrm{F} 170, \mathrm{I}$ have found $\left[\frac{\mathrm{Fe}}{\mathrm{H}}\right]_{\odot}^{\mathrm{F} 170} \simeq-0.18$. Other elements have a similar slight deficiency.

The effective temperature of F 244 deduced from $R-I$ implies inconsistent results. Note that this star is misplaced in the colour-colour diagram of the DDO photometry. Therefore, I have determined its atmospheric parameters independently from the photometric data, drawing a polygonal diagram as described in Blanc-Vaziaga et al. (1973). A satisfying model could be $\theta_{\text {eff }}=1.20$ and $\log g=1.70$; the resulting abundance is $\left[\frac{\mathrm{Fe}}{\mathrm{H}}\right]{ }_{\odot}^{\mathrm{F} 244} \simeq-0.02$.

So the mean abundance $I$ find for these stars in $M 67$ is -0.10 : let us say that $M 67$ has a solar metal content: from my analysis, it cannot be super metal-rich; the large absorption measured by Spinrad and Taylor (1969) in M 67 stars has to be interpreted in other terms than in terms of global overabundance of $M 67$ stars relative to the sun. 
I would want to conclude by emphasizing implications of these analyses about the chemical evolution of our Galaxy. First, the rather young cluster of Hyades has a solar metal content. Second, the old galactic cluster M 67 has also a solar metal content. This would support the idea that there is no significant metal enrichment in the galactic disk since the birth of $\mathrm{M} 67$, about $7.10^{9}$ ago. This conflicts with the recently proposed models of chemical evolution of the galactic disk, such as that by Pagel or Tinsley.

\section{References}

Blanc-Vaziaga, M.-J., Cayrel, G., and Cayrel, R.: 1973, Astrophys. J. 180, 871.

Spinrad, H. and Taylor, D. J.: 1969, Astrophys. J. 157, 1279.

\section{DISCUSSION}

Williams: First, a comment: the mean Hyades giant star abundance you give includes abundances of several stars assigned to the Hyades kinematic group - whose membership is controversial. The mean abundance of the three Hyades cluster giants you give is $[\mathrm{Fe} / \mathrm{H}]=0.17$, close to the canonical value.

Secondly, what damping do you use for the $\mathrm{Mg} b$ lines?

Foy: (1) I do not think that $[1 \mathrm{e} / \mathrm{H}]=+0.17$ is significantly different from $[\mathrm{Fe} / \mathrm{H}]=+0.06$.

(2) I use the damping constant resulting from the fit of the theoretical profile for the Sun to the observed one. This is therefore independent from the theory of damping.

Cayrel: Even if the Hyades giants have: $[\mathrm{Fe} / \mathrm{H}] \sim+0.2$ the important fact is that the Hyades dwarfs have: $[\mathrm{Fe} / \mathrm{H}] \sim 0$.

Osborn: You have given in your slides values of surface gravity and effective temperature for these stars. Have you checked these values by computing masses for these stars using the distance modulus?

What are the masses you derive?

Foy: Yes, I have. Masses would lie between 0.2 and 0.8 or $1.0 M_{\odot}$, depending upon the adopted distance modulus.

Martinet: In your statement on the non-enrichment of the galactic disc, you implicitly assume that NGC 188 and M 67 are typical, representative of objects of their age. On the contrary, one could consider these clusters as peculiar (long-lived amongst the open clusters) objects. Your statement may be correct but ought to be proved with the help of substantial material including objects well spread in age and in possible regions of formation. Further, NGC 2420 could be a counter-example.

Foy: I do not know if the dynamical peculiarity of M 67 can be related to the chemical composition of the interstellar matter which has generated it.

In our group in Meudon, we have analysed a lot of stars which are old and have a normal metal content.

It will be interesting to perform detailed analyses of stars in NGC 2420 .

Gustafsson: You concluded from your results for the solar-type dwarfs that scaled Peytremann models may be used with confidence for the giants. I am not sure you are quite correct in that conclusion since Peytremann did not include molecular lines which are of some importance for the temperature structures of the giants (as is shown by our models) but not for the solar-type dwarfs.

Foy: We have investigated with G. Cayrel and T. Tsuji the region of the $\log g / T_{\text {eff }}$ plane where molecular blanketing affects the $T(\tau)$ law in the line formation optical depths (Foy, 1974, thesis).

Models used in the present study fall in the 'good' region of this plane (except, maybe, in the case of $\alpha \mathrm{Tau}$ ): effects of molecular blanketing do not significantly affect the derived abundances.

Obviously, if both atomic and molecular blanketed models, as your ones, had been available when I started this work, I would have used them. 
Nissen: Your determination of the iron abundance of the two Hyades $\mathrm{G}$ dwarfs seems convincing, because you measured the equivalent widths of very weak lines, i.e. $W<30 \mathrm{~m} \AA$. Some of the discrepancy with the higher abundance obtained from photoelectric photometry may be explained if the Hyades have a peculiar high 'microturbulence'. Do you find any indication of this in your own electronographic data?

Foy: I think that the discrepancy between photometric and spectroscopic determination of Hyades abundance could come from the influence of microturbulence on photometric bands.

In the present work, I have mainly studied weak lincs, so, now, I have no indication of that from my data. But we are investigating this point with $R$. and G. Cayrel.

Bell: How do you explain the ultra-violet excess of the Sun relative to the Hyades main sequence, if the Sun has the same abundance as the Hyades?

Earlier suggestions by Conti and Deutsch that high $\delta m_{1}$ values could be produced by high Doppler Broadening Velocities have never been confirmed spectroscopically.

Foy: (1) Colours of the Sun are not known with enough accuracy to be really sure of this excess. It would be related to a higher microturbulent velocity in Hyades dwarfs.

(2) Accurate measurements of Doppler Broadening Velocities is very delicate in dwarfs, because of the large differences in damping constants - this is very well shown on my iron curve of growth of the Sun (Foy, 1972), of which the 'flat' part is strongly split.

Mayor: The difference that you obtain between Hyades and M 67 metallicities is $0.15 \pm 0.30$ (?) so from this, one can estimate a difference of about $\Delta[\mathrm{Fe} / \mathrm{H}]=0.3 \pm 0.6$ in $10^{10} \mathrm{yr}$. It seems to me that this fact is not a proof against a galactic enrichment.

Foy: I do not say that my results prove that there is no metal enrichment in the galactic disc, but that they support this idea. 